\title{
The effects of graphemic, phonetic, and semantic relationships on access to lexical structures
}

\author{
HARVEY G. SHULMAN, ROSEMARY HORNAK, and ELIZABETH SANDERS \\ Ohio State University, Columbus, Ohio 43210
}

\begin{abstract}
The question of whether or not lexical information is accessed directly from a visual code or by a process of phonetic mediation was investigated in three lexical decision experiments. Phonetic similarity influenced decisions about visually presented words only when they were to be discriminated from orthographically regular nonwords. When consonant strings or random letter strings were used as nonwords, phonetic similarity effects were absent, and graphemic similarity exerted a powerful effect while evidence of semantic priming was found. This pattern was interpreted as evidence of direct lexical access, which is probably the normal processing mode for skilled readers. Phonetic coding, when it occurs, may be a storage strategy rather than a part of the addressing chain for lexical structures.
\end{abstract}

Reading a visually presented word might involve any of several possible processing sequences in order to make contact between the stimulus and its stored representation. Each of these sequences must begin with the coding of visual features and end with the retrieval of semantic content. The nature of the processes occurring between these endpoints has been a topic of recent theoretical interest (Bradshaw, 1975) and has provided the central concern of the research reported in this paper.

Of particular concern to us was the role of phonetic codes. Are such codes a necessary mediating step between visual and semantic coding? Intuition can be used to support either position on this issue. On the one hand, reading involves mapping a visual pattern onto linguistic concepts originally acquired in the course of speaking and hearing. On the other hand, silent reading can be so rapid that the possibility of direct access from orthographic to semantic codes must be entertained.

Just as intuition provides no certain guide, so the recent literature can be used to support either the direct or mediated access hypothesis. The direct access view was supported in research reported by Frederiksen and Kroll (1976). They showed that certain variables related to the length and structure of a letter string influenced the time needed to pronounce the string, but not the time needed to make a lexical decision. The lexical decision task requires silent reading of the

This research was supported by NSF Grant BMS75-03867 to the first author. Reprint requests may be sent to Harvey G. Shulman, Human Performance Center, 404-B West 17th Avenue, Columbus, Ohio 43210 . We wish to thank David Meyer for providing a complete listing of the stimulus materials used by Meyer, Schvaneveldt, and Ruddy (1974). letter string followed by a word-nonword classification response. If the requirement to access the internal lexicon during silent reading involves the same kind of phonetic coding as does pronouncing a letter string, then both should be influenced in similar ways by variables such as string length and structure. In another instance, Forster and Chambers (1973) reached the conclusion that lexical access is not necessarily preceded by phonetic coding. Like Frederiksen and Kroll, they did this by arranging a comparison between naming and lexical decision tasks. The interpretation of these experiments rests on the assumption that naming and lexical decision differ, at most, by the inclusion of a phonetic coding step prior to lexical access in the naming task. While this assumption can be defended, it is not necessarily compelling, so uncertainty remains about the direct access hypothesis.

The mediated access hypothesis, that phonetic coding intervenes between graphemic and semantic coding, gains support in several experiments that did not rely on comparisons across different types of task. Rubenstein, Lewis, and Rubenstein (1971) found, in a lexical decision experiment, that negative response times increased as a direct function of nonword pronounceability. While this finding is consistent with the phonetic coding model, Meyer, Schvaneveldt, and Ruddy (1974) pointed out that it is also consistent with the direct access model, because nonwords constructed to be more pronounceable also conform more closely to the orthographic structure of words. So the Rubenstein et al. results may reflect differences in the ease of graphemic coding without implying that phonetic coding occurred in their task.

Meyer, Schvaneveldt, and Ruddy (1974) circumvented this problem by manipulating orthographic and phonemic relationships among words orthogonally 
in a lexical decision task. Their data also support the phonetic coding hypothesis, in what seems a convincing way. Since our experiments involve replicating and extending their procedures, a description of their method will serve as well to introduce our experiments. And since our data include a replication of their results, a description of ours will serve as well for theirs.

On each trial subjects were presented with two letter strings simultaneously and asked to respond "yes" if both were words and "no" if one or both were nonwords. The most important comparisons involved word pairs of four types, differing in the graphemic and phonetic similarity between members of each pair. Type 1 involved homophonic pairs, such as BRIBE-TRIBE, which were similar both graphemically and phonetically. Type 2 were control pairs for Type 1 and were constructed by randomly interchanging the first members of Type 1 pairs, with the result that Type 2 pairs were graphemically and phonetically dissimilar. Type 3 pairs were constructed from graphemically similar heterophones, pairs with dissimilar pronunciations (e.g., FREAK and BREAK). Type 4 pairs were control pairs for Type 3 , constructed by interchanging first members of Type 3 pairs. The logic of this design is based on the expectation that, if phonetic properties are not coded, Type 1 and Type 3 pairs should be functionally identical, and responded to more rapidly than Types 2 and 4 , which lack graphemic similarity. In contrast, if phonetic coding does play a role, Type 1 and Type 3 pairs would not be expected to function identically. The phonetic similarity of Type 1 pairs, for example, might increment the facilitation due to graphemic similarity, while the phonetic dissimilarity of Type 3 pairs might act against the facilitation due to graphemic similarity. In short, if phonetic properties are coded during lexical access, responses to Type 1 pairs should be faster than to Type 2 controls; this difference should be reduced or reversed in the comparison between Type 3 and their Type 4 controls. This is exactly what Meyer, Schvaneveldt, and Ruddy (1974) found, and their experiment can be viewed as a demonstration of mediated access.

While Meyer, Schvaneveldt, and Ruddy's (1974) experiment may demonstrate that mediated access can occur, it provides no answer to the question of whether mediated access is the only processing mode possible. To illustrate our reason for believing that direct access may be available as an option, consider a recent experiment by Shulman and Davison (1977), which was concerned with the effects of semantic relatedness on lexical decisions. Meyer and Schvaneveldt (1971) reported that lexical decisions about pairs of words were facilitated by semantic relatedness between pair members. Shulman and Davison (1977) showed that the magnitude of this effect depended upon the nonword context from which word pairs were to be discriminated. Using orthographically regular, pronounceable letter strings as nonwords (we will refer to such strings as pseudowords), the semantic relatedness effect was about $100 \mathrm{msec}$. When consonant strings were used as nonword items, however, the relatedness effect was reduced to about $30 \mathrm{msec}$. This reduction was interpreted as reflecting a shift in processing mode, made possible by the changed requirements of the word-nonword discrimination and the optional nature of semantic coding.

Meyer, Schvaneveldt, and Ruddy's (1974) experiment required that words be discriminated from pseudowords. The question addressed by our first two experiments was whether or not the effects of phonetic similarity on decisions about word pairs might be eliminated or reduced by using phonetically and orthographically illegal letter strings as nonwords. We predicted that, if direct access was possible under these conditions, Type 1 (BRIBE-TRIBE) and Type 3 (FREAK-BREAK) word pairs would be functionally identical. The experiments reported include replications of the Meyer, Schvaneveldt, and Ruddy (1974) experiment using pseudowords as negative items. Under these conditions we expected to replicate their finding of facilitation for Type 1 as compared to Type 3 pairs.

\section{EXPERIMENT 1}

\section{Method}

Design. Two groups of 24 Ohio State University students served as subjects in order to fulfill a course requirement. They performed a lexical decision task, in which two simultaneously presented letter strings were responded to positively if both were words or negatively if one or both were nonwords The two groups differed from one another only in the nature of the nonword items presented. For one group these were pseudowords constructed by replacing consonants with consonants, or vowels with vowels, in familiar English words, in such a way that they became nonwords. For the other group the nonwords were consonant strings, formed by replacing consonants for the vowels in the pseudowords used for the first group.

For each subject an initial block of 72 practice trials was followed by 3 blocks of 72 experimental trials, upon which subsequent data analyses were based. In each block 32 word pairs were presented, comprised of eight graphemically and phonemically similar Type 1 pairs (e.g., BRIBE-TRIBE, MIGHTTIGHT), eight control pairs constructed by rearranging Type 1 pairs (e.g., BRIBE-TIGHT, MIGHT-TRIBE), eight graphemically similar, phonetically dissimilar Type 3 pairs (e.g., FREAK BREAK, COUCH-TOUCH), and eight Type 4 control pairs derived from Type 3 pairs (e.g., FREAK-TOUCH, COUCHBREAK). The remaining 40 trials in each block included 16 graphemically similar pairs composed of a word and a nonword, 16 graphemically dissimilar word-nonword pairs, 4 graphemically similar pairs composed of two nonwords (Type NN), and 4 graphemically dissimilar pairs composed of two nonwords. Half of the word-nonword pairs were presented with the word in the top display position, half with the word in the bottom position. These will be referred to as word-nonword (Type WN) and nonword-word pairs (Type NW), respectively. Within each block the order of presentation of the various pair types was randomly determined, with a new sequence generated for each experimental session.

Materials. For each subject the stimuli used in three experi- 
mental trial blocks were drawn from one of eight lists, each composed of 216 pairs of letter strings, ranging in length from three to nine letters. Of the eight lists, there were two sets of four which differed from one another only in the type of nonword used (pseudowords or consonant strings). Each list contained 24 exemplars of Types 1,2,3, and 4 word pairs, 12 exemplars each of the two types of nonword-nonword pairs, and 24 exemplars each of the four types of wordnonword pairs. The words and pseudowords used were taken from Meyer. Schvaneveldt, and Ruddy (1974). ${ }^{1}$

The four lists used in each nonword context condition were derived from each other by rearranging items between pairs assigned to different experimental conditions. This was done to achieve a partial crossing of stimuli and conditions. The crossing was only partial because the relationship between stimuli defined the conditions and some stimuli could not be used in certain conditions. Instead, individual stimuli were nested within Types 1 and 2, Types 3 and 4 , and within the various nonword conditions. To accomplish this nesting, two lists were needed. The additional two lists in each set of four were derived from the original two by exchanging the display positions (top or bottom) of the pair members.

No subject saw the same letter string more than once in the experiment. The design described above provides for sets of four subjects in which the assignment of stimuli to conditions was balanced by presenting each subject with one of the four lists described above. By collapsing data over such sets of four subjects, we created pseudosubjects who had experienced each letter string as a member of a graphemically similar and a graphemically dissimilar pair. For each pseudosubject the stimuli were partitioned into three subsets, and the data within each subset were averaged separately for each experimental condition. These procedures were sufficient to insure that subsequent analyses could treat subjects, word sets, and experimental conditions as unconfounded effects.

Procedure. The experiment was conducted using a small CRT display controlled by a NOVA computer. Each trial began with the presentation of a ready signal (five Xs) for $1 / 2 \mathrm{sec}$, followed after a $1 / 2$-sec blank interval by the presentation of a pair of letter strings, drawn in capital letters. The two strings were presented, one above the other, for 2 sec; subjects pressed one button if the strings were both words, another if one or both were nonwords. Following stimulus offset, a 2 -sec intertrial interval commenced, during which an error message was presented if warranted. At the end of each block of trials, the experimenter informed the subject of his median reaction time (RT) and total number of errors for that block Subjects were instructed to attempt to make no more than two or three errors per block, and to respond as quickly as the error constraint would allow.

\section{Results}

Analyses of variance were done to analyze RT and error data, separately for the word-word conditions and the nonword conditions. Trials on which erroneous responses were made, or responses made after a 3 -sec deadline, were excluded from the analyses. In the analyses to be reported, subsets of stimuli were treated as a fixed effect, since our sampling procedures could not be considered random, or even quasirandom. However, additional analyses were also done in which stimuli were treated as a random effect. The two sets of analyses showed essentially the same pattern of effects. In the results reported in Table 1, all statistical tests were evaluated at the .05 alpha level.

The data for word pairs are shown in Table 1. The
Table 1

Mean Reaction Time (RT) and Percent Errors (\%E) for Word Pairs in Experiment 1

\begin{tabular}{ccccccc}
\hline & & \multicolumn{3}{c}{ Nonword Context } \\
\cline { 5 - 7 } Type & Example & R T & $\% \mathrm{E}$ & & RT & $\% \mathrm{E}$ \\
\hline 1 & & BRIBE-TRIBE & 1069 & 5.1 & 649 & .5 \\
2 & BRIBE-TIGHT & 1123 & 3.3 & 787 & 3.9 \\
3 & FREAK-BREAK & 1179 & 11.1 & 643 & 1.0 \\
4 & FREAK-WORSE & 1094 & 4.8 & 744 & 2.4 \\
\hline
\end{tabular}

Note-Reaction times are based on correct responses only.

column headings identify the nonword context, that is, the kind of nonwords from which the word pairs were to be discriminated. These data were analyzed in a partially hierarchical design with stimulus subsets nested under phonetic similarity conditions and crossed with all other factors. Within this design, planned comparisons were used to test the differences between Type 1 and 2 pairs, and between Types 3 and 4 . When pseudowords were used as negative items, the data provided a replication of Meyer, Schvaneveldt, and Ruddy's (1974) results. Responses to Type 1 pairs were reliably faster $(54 \mathrm{msec})$ than were their Type 2 controls $[F(1,10)=25.38, \mathrm{MSe}=1,034]$, while Type 3 pairs were reliably slower $(85 \mathrm{msec})$ than Type 4 $[\mathrm{F}(1,10)=62.89, \mathrm{MSe}=1,034]$. This pattern can only be a consequence of the difference in the phonetic relationship between Type 1 and Type 3 pairs.

An entirely different pattern emerged when consonant strings instead of pseudowords were used as negative items. Responses to the word pairs in this nonword context were reliably faster than to the same word pairs when they were discriminated from pseudowords $[F(1,10)=98.75, \quad M S e=61,446]$. Planned comparisons showed that Type 1 and Type 3 pairs were both reliably faster than their Type 2 and 4 controls, $F(1,10)=165.76, \mathrm{MSe}=1,034$ for Type 1 vs. Type 2, and $F(1,10)=88.79$, MSe $=1,034$ for Type 3 vs. Type 4 . The decreases in RT for the two similar types of word pairs relative to their controls (138 $\mathrm{msec}$ for Types 1 and 2, $101 \mathrm{msec}$ for Types 3 and 4) were not reliably different from each other.

Both the effects of nonword context and of word pair type entered into interactions with stimulus subsets in the RT data for word pairs. A reliable two-way interaction occurred between nonword context and stimulus subset $[F(4,40)=4.72, \quad M S e=2,783]$, as did a three-way interaction between type of word pair, stimulus subset, and nonword context $[F(4,40)=$ $2.85, \mathrm{MSe}=1,513]$. These interactions were due to variability in the size, but not the direction, of differences between conditions in the three stimulus subsets.

Table 2 shows the RT data for nonword pairs in 
Table 2

Mean Reaction Time (RT) and Percent Errors (\%E) for Negative Pairs in Experiment 1

\begin{tabular}{|c|c|c|c|c|c|c|c|c|}
\hline \multirow[b]{4}{*}{ Type } & \multicolumn{8}{|c|}{ Nonword Structure } \\
\hline & \multicolumn{4}{|c|}{ Pseudowords } & \multicolumn{4}{|c|}{ Consonant Strings } \\
\hline & \multicolumn{2}{|c|}{ GS } & \multicolumn{2}{|c|}{ GD } & \multicolumn{2}{|c|}{ GS } & \multicolumn{2}{|c|}{ GD } \\
\hline & RT & $\% \mathrm{E}$ & RT & $\% \mathrm{E}$ & RT & $\% \mathrm{E}$ & $\mathrm{RT}$ & $\% \mathrm{E}$ \\
\hline WN & 1168 & 6.4 & 1290 & 7.3 & 791 & 10.2 & 773 & 5.7 \\
\hline NW & 1122 & 4.3 & 1118 & 4.9 & 664 & 2.6 & 640 & .7 \\
\hline NN & 1071 & .4 & 1124 & 0.0 & 613 & .4 & 625 & .4 \\
\hline
\end{tabular}

Note-Reaction times are based on correct responses only. $G S=$ graphemically similar; $G D=$ graphemically dissimilar.

each experimental condition. Consonant strings were responded to more rapidly than pseudowords $[F(1,10)=$ 92.66, MSe 125,766], and this effect interacted with the graphemic similarity of the pairs $[F(1,10)=$ $9.09, \mathrm{MSe}=59,564]$. This interaction reflects the fact that graphemic similarity affected RT to nonword pairs constructed with pseudowords, but not with consonant strings. The type of nonword pair (WN, NW, or NN) also affected RT $[\mathrm{F}(2,20=178.06$, MSe $=2,480]$, which simply reflects the fact that pairs with a word in the top display position (WN) took longest to discriminate from word pairs, and pairs with no word (NN) took the least time.

The average percentages of errors made in each condition are shown in Tables 1 and 2. For word pairs the overall error percentage was 4.0 , and for nonword pairs, 4.3. The error rate for word pairs was reliably affected by nonword context $[\mathrm{F} 1,10)=84.96$, MSe $=$ $.045]$, type of pair $(1,2,3$, or 4$)[F(3,30)=7.46$, $\mathrm{MSe}=.061]$, and the interaction of these factors $[\mathrm{F}(3,30)=19.67, \mathrm{MSe}=.061]$. In general, these effects paralleled the pattem observed in the RT data, with those conditions having the longest RTs also having higher error rates. The exception was for Type 1 and 2 word pairs where the error rate was higher for Type 1 than Type 2. In the nonword error data, two main effects and four interactions were reliable. These main effects were graphemic similarity $[F(1,10)=16.42$, $\mathrm{MSe}=.016]$ and pair type $(\mathrm{WN}, \mathrm{NW}, \mathrm{NN})[\mathrm{F}(2,20)=$ $54.16, \mathrm{MSe}=.114]$. These effects are difficult to interpret since each entered into a reliable two-way interaction with nonword structure and a reliable three-way interaction with each other and nonword structure $[\mathrm{F}(2,20)=7.89, \mathrm{MSe}=.028]$.

\section{Discussion}

While a complete discussion of the data will be postponed until the results of Experiments 2 and 3 are reported, some comments on the major findings of Experiment 1 are needed at this point. The most important finding of Experiment 1 is that phonetic properties of the word pairs influenced lexical decisions only when words were to be discriminated from pseudo- words having the orthographic and phonetic structure of English. When words and nonwords were discriminable on the basis of graphemic and phonetic properties, processing of phonetic features apparently was abandoned, and graphemic relationships between the letter strings exerted a powerful effect.

There are at least two ways to interpret this pattern of results. First, direct access to lexical information without phonetic mediation may be possible but optional, with the nature of the nonword context influencing the processing path chosen for words. Taken in this light, our data indicate that words and nonwords were discriminated from one another on the basis of a test of lexical content, with phonetically mediated access used for words and nonwords when psuedowords were the negative items, but with direct access used when consonant strings were negative items. A second explanation is that, while phonetically mediated lexical access was used to discriminate words from pseudowords, a truncated processing mode may have been used in the consonant string conditions, with words and nonwords discriminated on the basis of a test for graphemic regularity. Our data are consistent with this possibility, and viewed in this way they provide no support for the direct access model. The simplest way to discriminate words from consonant strings without lexical access would be to search for the presence of a vowel anywhere in the stimulus. Experiment 2 was designed to preclude this possibility, by using random letter strings instead of consonant strings as nonwords. These strings were constructed by randomly permuting the letters in pseudowords, so that the resulting strings violated English orthography without omitting vowels.

\section{EXPERIMENT 2}

\section{Method}

Design. Experiment 2 was a replication of Experiment 1, with random letter strings replacing consonant strings as the nonwords for one group of 24 subjects. The second group of 24 subjects was presented with pseudowords as nonword items. The 48 subjects were recruited from the same population as those who served in Experiment 1, although no subject served in both experiments. The procedures, materials (except for the random strings), and data reduction procedures were also identical to Experiment 1.

\section{Results}

The data for word pairs are shown in Table 3. The RT data replicate the results of Experiment 1, showing an effect of phonetic similarity when words were discriminated from pseudowords, and a substantial decrease in RT coupled with a powerful graphemic similarity effect when words were discriminated from random letter strings. As in Experiment 1, planned comparisons were used to evaluate the differences between Type 1 and Type 2 pairs and also between Types 3 and 4 , separately for the pseudoword and random string conditions. In both nonword contexts Type 1 pairs 
Table 3

Mean Reaction Time (RT) and Percent Errors (\%E) for Responses to Word Pairs in Experiment 2

\begin{tabular}{|c|c|c|c|c|c|}
\hline \multirow[b]{3}{*}{ Type } & \multirow[b]{3}{*}{ Example } & \multicolumn{4}{|c|}{ Nonword Context } \\
\hline & & \multicolumn{2}{|c|}{ Pseudowords } & \multicolumn{2}{|c|}{$\begin{array}{c}\text { Random } \\
\text { Strings } \\
\end{array}$} \\
\hline & & RT & $\% \mathrm{E}$ & RT & $\% \mathrm{E}$ \\
\hline 1 & FIGHT-TIGHT & 982 & 4.2 & 691 & .5 \\
\hline 2 & FIGHT-TRIBE & 1020 & 3.7 & 817 & 3.1 \\
\hline 3 & HORSE-WORSE & 1092 & 7.9 & 697 & .9 \\
\hline 4 & HORSE-BREAK & 1007 & 2.8 & 792 & 2.4 \\
\hline
\end{tabular}

Note-Reaction times are based on correct responses only.

were reliably faster than Type $2[F(1,10)=14.41$, MSe $=902$ for the pseudoword context, and $F(1,10)=$ $158.41, \mathrm{MSe}=902$ for the random string conditions] . Type 3 and 4 word pairs were also reliably different from one another in both nonword contexts, but, as in Experiment 1, the differences were in opposite directions. In the pseudoword context, Type 3 pairs were responded to $85 \mathrm{msec}$ slower than were Type 4 pairs $[\mathrm{F}(1,10)=72.09$, MSe $=902]$, while in the context of random letter strings, they were judged faster than Type 4 pairs by $95 \mathrm{msec}[\mathrm{F}(1,30)=90.04$, $\mathrm{MSe}=902 \mathrm{l}$. As in Experiment 1, the decreases in RT for the two similar pair types relative to their controls in the random string condition (Type 1 - Type 2 vs. Type 3 - Type 4) were not reliably different from one another. Also significant in the data for word pairs were the main effects of nonword context $[F(1,10)=41.50$, $\mathrm{MSe}=66,090]$ and stimulus subset $[\mathrm{F}(4,40)=6.47$, MSe $=2,920]$. The latter effect was due to variation over word sets in speed of responding, which did not interact with any other variable.

The RT data for nonword pairs are shown in Table 4. The pattern of effects in these data is quite similar to that in Experiment 1. However, the pattern of statistical tests was a good deal more complex in Experiment 2. Significant main effects of nonword structure (pseudowords vs. random strings) $[\mathrm{F}(1,10)=49.71, \mathrm{MSe}=$ $146,653]$, graphemic similarity $[\mathrm{F}(1,10)=9.39, \mathrm{MSe}=$ $2,218]$, and nonword pair type (WN, NW, NN) $[\mathrm{F}(2,20)$ $=110.13, \mathrm{MSe}=5,108 \mathrm{]}$ were found. In addition, all of the two-way interactions and the triple interaction involving these factors were also reliable. The main effects are straightforward. Reaction times to pairs containing one or two pseudowords were longer than to pairs containing random strings, pairs constructed of two nonwords were responded to more rapidly than those in which a word was present, and graphemically similar pairs were responded to more rapidly than graphemically different pairs, although the latter effect clearly occurred only with pseudowords. The triple in teraction reflected both this last fact and the fact that the size of the graphemic similarity effect depended upon pair type. These interactions probably reflect differences in the processing strategies and kinds of graphemic similarity in the various conditions. For example, pairs of Type NN and NW can be classified correctly by processing the top string in the display, while both members of Type WN pairs must be processed if we assume that processing starts with the top member of each pair. Graphemic similarity effects can only occur when both pair members are processed, so a pattern of interactions involving pair type and graphemic similarity is not surprising. The absence of graphemic similarity effects in the random string conditions, and also in the consonant string conditions of Experiment 1, may be due to a difference in the kind of graphemic similarity used in those conditions as compared to pseudoword conditions. Specifically, with WN or NW pairs it is possible to make the word and nonword similar in both letter content and letter order when pseudowords are used (e.g., NIZE, SIZE), but when random strings or consonant strings are used, one or the other of these constraints must be relaxed (e.g., ZNEI, SIZE or SLZF, SIZE). The fact that violation of order similarity as well as violation of item similarity can attenuate graphemic similarity effects can be taken as evidence that the orthographic code generated in our task contained order information as well as item information, and that graphemic priming effects are based on this code rather than on item information alone.

The error data for Experiment 2 are shown in Tables 3 and 4 . The overall error rate for word pairs was $3.2 \%$, and $3.5 \%$ for nonword pairs. For word pairs there were reliable main effects of nonword context $[\mathrm{F}(1,10)=27.47, \mathrm{MSe}=.071]$, word pair type $[\mathrm{F}(3,30)=3.10$, MSe $=.065]$, and an interaction between the two $[F(3,30)=9.00$, MSe $=.065]$. These results parallel the RT data, with longer RTs associated with larger error rates in all instances except for the comparison of Type 1 and 2 pairs in the pseudoword context.

For nonword pairs the only significant main effect in the error data was pair type $[\mathrm{F}(2,20)=58.31$, MSe $=.057]$. This main effect interacted with nonword structure $[\mathrm{F}(2,20)=11.41, \mathrm{MSe}=.057]$; in addition, there was an interaction in the error data

Table 4

Mean Reaction Time (RT) and Percent Errors (\%E) for Negative Pairs in Experiment 2

\begin{tabular}{|c|c|c|c|c|c|c|c|c|}
\hline \multirow[b]{4}{*}{ Type } & \multicolumn{8}{|c|}{ Nonword Structure } \\
\hline & \multicolumn{4}{|c|}{ Pseudowords } & \multicolumn{4}{|c|}{ Random Strings } \\
\hline & \multicolumn{2}{|c|}{ GS } & \multicolumn{2}{|c|}{ GD } & \multicolumn{2}{|c|}{ GS } & \multicolumn{2}{|c|}{ GD } \\
\hline & RT & $\% \mathrm{E}$ & RT & $\% \mathrm{E}$ & RT & $\% \mathrm{E}$ & RT & $\% \mathrm{E}$ \\
\hline WN & 1046 & 5.0 & 1136 & 5.2 & 734 & 6.3 & 718 & 5.7 \\
\hline NW & 1082 & 3.8 & 1117 & 5.6 & 730 & 1.4 & 700 & .9 \\
\hline NN & 1056 & 0.0 & 1092 & .4 & 720 & 1.0 & 724 & 0.0 \\
\hline
\end{tabular}

Note-Reaction times are based on correct responses only. $G S=$ graphemically similar; $G D=$ graphemically dissimilar. 
between nonword structure, graphemic similarity, and stimulus subset $[\mathrm{F}(2,20)=5.13$, MSe $=.073]$.

\section{Discussion}

Experiment 2 was conducted in order to determine which of two processing modes, direct lexical access or truncation prior to phonetic coding, was responsible for the effect of nonword structure on word-nonword discrimination. Either of these modes was consistent with the results of Experiment 1 (replicated in Experiment 2) which showed that RT and error rates were influenced by phonetic similarity only when words were to be discriminated from orthographically legal (hence pronounceable) nonwords. Since words can logically be discriminated from consonant strings simply by searching for vowels, it was possible that a very primitive level of graphemic coding might provide information sufficient to permit termination of processing and selection of the correct response. In this case, phonetic similarity effects would not occur, RT would be faster, and graphemic similarity might have a strong effect on decision times.

The random letter strings used as orthographically illegal nonwords in Experiment 2 approximated words in terms of consonant and vowel frequencies. Since the results of Experiment 2 replicate those of Experiment 1, it appears that no simple strategy of searching for or counting vowels can explain the dependence of phonetic similarity effects on nonword context: Phonetic similarity affected word judgments only when the word pairs were to be discriminated from pseudowords. While these results are consistent with the direct access model, it is still possible that a truncated processing mode may have been used, with truncation after the formation of a relatively high-level orthographic code. This possibility is suggested by our nonword data in which graphemic similarity affected RT for words and pseudowords but not for orthographically irregular strings. One might expect that, if the graphemic priming effect is based on a high-level orthographic code (e.g., vocalic center groupings), then priming might not occur with letter strings that are difficult to parse into such codes. Still, the fact that such codes may have been used by our subjects is not sufficient evidence to choose between truncation and direct lexical access. The unanswered question remains: When words were to be discriminated from orthographically illegal letter strings, was processing terminated prior to phonetic coding and lexical access, or was phonetic coding omitted but lexical access attempted?

To resolve this question, evidence is required which implicates lexical involvement at the same time that phonetic coding is shown not to occur. Experiments 1 and 2 demonstrate that the latter condition can be met by requiring a discrimination between words and orthographically irregular letter strings. In order to probe for lexical involvement under these conditions, we sought to determine whether the semantic relationship between the members of a word pair would influence lexical decisions. In a recent experiment by Shulman and Davison (1977), we demonstrated such effects when words were discriminated both from pseudowords and from consonant strings. The semantic priming effects (Meyer \& Schvaneveldt, 1971) found were large in the word-pseudoword discrimination and small but reliable in the word-consonant string discrimination. In Experiment 3 semantically related word pairs along with pairs of Types $1,2,3$, and 4 from Experiments 1 and 2 were discriminated from pairs including one or two random letter strings. If the truncation model is correct, semantically related pairs should be responded to no differently than control pairs. In order to validate the direct access model, it is necessary for pairs of Type 1 (FAWNDAWN) and Type 3 (HOSE.LOSE) to be functionally identical, verifying the absence of phonetic coding, while pairs of semantically related words are responded to more rapidly than control pairs, indicating lexical involvement.

\section{EXPERIMENT 3}

\section{Method}

The experimental procedures were identical to those of the previous two experiments. Experiment 3 differed in its design in that a single group of 48 subjects, recruited as before, served in a lexical decision task requiring discrimination between word pairs and pairs constructed with at least one random letter string. The stimulus materials from the random letter string condition of Experiment 2 were supplemented by an additional set of 48 semantically associated word pairs, selected from those used by Shulman and Davison (1977). These pairs were divided into two sets of 24 pairs, and from each a set of 24 unrelated pairs was constructed by rearranging pair members. Four related pairs from one set of 24 and 4 unrelated pairs derived from the other set of 24 related pairs were then incorporated in each of the six experimental trial blocks. As a consequence, in each block of trials there occurred four graphemically similar, phonetically similar Type 1 pairs, four dissimilar Type 2 pairs, four graphemically similar, phonetically dissimilar Type 3 pairs, four dissimilar Type 4 control pairs, four semantically associated pairs (Type 5), and four semantically unrelated control pairs (Type 6). For each subject the control pairs for each similarity condition were derived from the experimental pairs seen by another subject. The nonword conditions were exactly the same as in the first two experiments.

\section{Results}

The data reduction procedures used in Experiment 3 resembled those used in Experiments 1 and 2 in that we averaged median RTs over subsets of four subjects, chosen so that the resulting pseudosubjects had experienced each stimulus as a member of both a related and an unrelated control pair. A minor difference between Experiment 3 and the earlier experiments was that we partitioned each set of stimuli into six 
Table 5

Mean Reaction Time (RT) and Percent Errors (\%E) for Responses to Word Pairs in Experiment 3

\begin{tabular}{cllr}
\hline Type & Example & RT & $\%$ E \\
\hline 1 & FIRE-HIRE & 624 & 1.3 \\
2 & FIRE-HAIR & 715 & 4.5 \\
3 & YOUTH-SOUTH & 625 & .5 \\
4 & YOUTH-LEMON & 692 & 2.1 \\
5 & STREET-ROAD & 664 & 1.9 \\
6 & STREET-ROCK & 707 & 3.3 \\
\hline
\end{tabular}

Note-Reaction times are based on correct responses only.

subsets, rather than three, and used the median RT and total number of errors for each of the six subsets as the basis for statistical tests of the generality of effects over subsets of stimuli. As before, these tests were done with stimuli as both fixed and random effects, with no important differences in the resulting pattern of statistical tests. The statistical tests reported are those in which stimuli were regarded as a fixed effect.

The data for correct responses to word pairs are shown in Table 5; as in Experiments 1 and 2, there appears to have been no functional difference between Type 1 and Type 3 pairs, and hence no evidence of phonetic encoding. Type 1 pairs were responded to $91 \mathrm{msec}$ faster than Type 2 pairs $[F(1,22)=115.87$, MSe $=$ 1,290], and Type 3 pairs were responded to $67 \mathrm{msec}$ faster than Type 4 control pairs $[F(1,22)=61.15$, $\mathrm{MSe}=1,290]$. Furthermore, the comparison of Type 5 (semantically associated) and Type 6 (semantically unrelated) pairs provides evidence for semantic priming $[\mathrm{F}(1,22)=26.28$, MSe $=1,290]$, in that associated pairs were responded to $43 \mathrm{msec}$ faster than unrelated pairs. In addition to these effects, RT for word pairs was reliably influenced by stimulus subset $[F(15,165)=$ $6.11, \mathrm{MSe}=3,086]$ and by the interaction of stimulus subset with type of word pair $[F(15,165)=3.27$, MSe $=1,154]$. This interaction again reflected variation in the size, but not the direction, of the experimental effects observed in the separate stimulus subsets.

In the RT data for nonword pairs, shown in Table 6 , there were reliable effects of pair type (WN, NW, NN) $[\mathrm{F}(2,22)=263.11, \mathrm{MSe}=5,698]$, graphemic similarity $[\mathrm{F}(1,11)=4.84, \mathrm{MSe}=3,058]$, and stimulus subset $[\mathrm{F}(15,165)=2.36, \mathrm{MSe}=39.5]$. The effect of graphe . mic similarity interacted with that of stimulus subset $[\mathrm{F}(15,165)=1.89, \mathrm{MSe}=1,304]$. This interaction was due to variations across subsets of stimuli in the magnitude and direction of the graphemic similarity effect, which on average was due to a 12 -msec advantage for graphemically dissimilar nonword pairs. We regard this effect as spurious, since nothing resembling it was found in either of our first two experiments.

The error data for responses to word pairs paralleled the RT data, in that comparisons between conditions revealed lower error rates for Type 1, 3, and 5 pairs as compared to their Type 2, 4, and 6 control pairs $[F(1,11)=42.43, \mathrm{MSe}=.017]$. The overall error rate for word pairs was $2.3 \%$. For nonword pairs the overall error rate was $6.9 \%$ and the only reliable experimental effect was pair type (WN, NW, NN) $[\mathrm{F}(2,22)=81.12$, $\mathrm{MSe}=.138]$.

\section{CONCLUSIONS}

Our primary concern has been with the question of whether access to lexical information may be accomplished directly from a graphemic code or must be phonetically mediated. The critical findings for answering this question come from those conditions in our experiments in which words were discriminated from orthographically irregular nonwords. These findings were that phonetic similarity between members of a word pair affected neither RT nor error rates, that semantic relationships did influence $\mathrm{RT}$, and that overall decision times were much shorter when words were discriminated from irregular nonwords rather than from pseudowords.

This pattern of results can be explained by the direct access hypothesis, which implies a model of lexical access in which a graphemic code forms the basis for addressing a semantically organized lexical structure. Semantic priming effects arise when successive retrieval attempts are made into the same region of lexical space. The basis for such priming effects is not the primary concern of this paper, so long as it is safe to assume that such effects imply the involvement of lexical structures in the word-nonword discrimination task. The "spreading activation" model proposed by Collins and Loftus (1975) is consistent with our observation of priming effects, but a number of other models might also be. Since phonetic information is not coded in the direct access model, RT should be shorter than when such codes are used. And effects of phonetic priming or phonetic confusion should not occur.

An alternative model for the effects observed when words were discriminated from irregular nonwords is based on the assumption that processing can be truncated prior to lexical access, and the word-nonword

Table 6

Mean Reaction Times (RT) and Percent Errors (\%E) for Negative Pairs in Experiment 3

\begin{tabular}{crrrrr} 
& \multicolumn{2}{c}{ GS } & & \multicolumn{2}{c}{ GD } \\
\cline { 2 - 3 } \cline { 5 - 6 } Type & RT & \%E & & RT & \%E \\
\hline WN & 812 & 14.5 & & 801 & 12.7 \\
NW & 644 & 3.1 & & 629 & 3.3 \\
NN & 627 & .9 & & 619 & .3 \\
\hline
\end{tabular}

Note-Reaction times are based on correct responses only. $G S=$ graphemically similar: $G D=$ graphemically dissimilar. 
decision made on the basis of graphemic or phonetic regularity. Since encoding of orthographic properties, those related to the visual information in the stimulus, must precede phonetic coding, it is easy to see how truncation after graphemic coding would preclude the occurrence of phonetic similarity effects. In the case of truncation after phonetic coding, an additional assumption is needed to explain the absence of phonetic effects observed in our experiments. This assumption is that words can be discriminated from irregular nonwords on the basis of phonetic regularity or ease of pronunciation without regard to whether the phonetic code generated was correct or in error for the presented word. Otherwise, we would expect to find that word pairs like FREAK-BREAK were responded to more slowly than dissimilar pairs when discriminated from irregular nonwords as well as pseudowords.

While either version of the truncation model can explain the dependence of phonetic similarity effects on nonword structure, neither seems compatible with the observation of semantic priming effects in the absence of phonetic effects, since semantic effects arise when lexical structures are accessed but not when processing stops short of lexical retrieval. Our data do not entirely preclude the possibility that a mixture of truncated processing and direct access was used in our experiments, but this conclusion too provides an affirmative answer to the question of whether or not direct access is possible. While the data demand the direct access assumption, they do not require the assumption of truncated processing.

Our data also include two replications of the phonetic similarity effects reported by Meyer, Schvaneveldt, and Ruddy (1974), when words were discriminated from orthographically and phonetically legal letter strings (pseudowords). Their interpretation of these effects was that lexical access requires phonetic mediation. Our data refute this conclusion, since we have shown that phonetic mediation is at most an option rather than a requirement. We are not sure, however, that the observed phonetic similarity effects, facilitation for word pairs like FIRE-HIRE, and inhibition for pairs like FREAK-BREAK necessarily imply that phonetic coding mediates between graphemic coding and lexical access. While these similarity effects indicate the involvement of phonetic information in wordpseudoword discrimination, there is no need to assign phonetic coding a role in addressing lexical structures.

In fact, the role played by phonetic information in our experiments is not easy to deduce. Phonetic similarity affected performance only when words were discriminated from pronounceable pseudowords. Yet words cannot be discriminated from pseudowords on the basis of phonetic regularity, and a test of lexical content is therefore required. Phonetic similarity did not affect performance in our experiments when words were discriminated from unpronounceable nonwords. Yet a test of phonetic regularity would do nicely here. The key perhaps is to recognize that words were coded in a manner dictated by the nonwords from which they were discriminated. When nonwords were pronounceable, words were phonetically coded; when nonwords were not pronounceable, words were not phonetically coded. In fact, up to the point of lexical access, words and pseudowords must be coded in the same way since wordness is indeterminate till then.

Our hypothesis is that the normal mode of lexical access is direct, not phonetically mediated, and that phonetic coding is introduced in the word-pseudoword discrimination task as a consequence of the difficulty in determining whether a pseudoword is a rare word or a nonword. The protracted search of memory induced by pseudowords might be facilitated by phonetic coding, perhaps as a way of holding information about the stimulus in short-term memory (STM) while directing queries into lexical memory. We assume that this strategy carried over to the processing of words, where pairs like FIRE-HIRE benefited from graphemic and phonetic priming, while pairs like FREAK-BREAK were slowed by the incorrect pronunciation assigned the second word processed. Because this phonetic coding process is primarily a storage aid in STM rather than a part of the addressing chain needed to access the lexicon, we do not consider it a true mediation process. This is a fine point to argue, however, and perhaps less important than the realization that the utility of phonetic coding stems from the processing requirements of nonwords rather than words. Orthographically irregular nonwords are difficult to parse phonetically and hence were probably not coded in this way. Thus, when words were discriminated from irregular nonwords in our experiments, no evidence of phonetic coding was obtained.

Although we find little support in our results for phonetic coding as a part of the process of addressing lexical information, we do not doubt that the cognitive system is flexible enough to accommodate such a process when it is necessary. The college students who served as our subjects are perhaps less likely to need phonetic mediation than a younger population of subjects would be, and developmental differences in phonetic mediation are an intriguing possibility. If the current procedures are to be used to explore such possibilities, however, it will be necessary to develop operations that can converge on the distinction between phonetic coding as a storage strategy and as an addressing or retrieval process.

\section{REFERENCES}

Bradshaw, J. L. Three interrelated problems in reading: A review. Memory \& Cognition, 1975, 3, 123-134. 
Collins, A. M., \& Lofrus, E. F. A spreading activation theory of semantic processing. Psychological Review, 1975, 82, 407-428.

Forster, K. I., \& Chambers, S. M. Lexical access and naming time. Journal of Verbal Learning and Verbal Behavior, 1973, 12, 627-635.

Frederussen, J. R., \& Kroll, J. F. Spelling and sound: Approaches to the internal lexicon. Journal of Experimental Psychology: Human Perception and Performance, 1976, 2, 361-379.

Meyer, D. E., \& Schvaneveldt, R. W. Facilitation in recognizing pairs of words: Evidence of a dependence between retrieval operations. Jourmal of Experimental Psychology, 1971, 90, 227-234.
Meyer, D. E., Schvaneveldt, R. W., \& Ruddy, M. G. Functions of graphemic and phonemic codes in visual word recognition. Memory \& Cognition, 1974, 2, 309-321.

Rubenstein, H., Lewis, S. S., \& Rubenstein, M. A. Evidence for phonemic recoding in visual word recognition. Journal of Verbal Learning and Verbal Behavior, 1971, 10 , 645-657.

Shulman, H. G., \& Davison, T. C. B. Control properties of semantic coding in a lexical decision task. Journal of Verbal Learning and Verbal Behavior, 1977, 16, 91-98.

(Received for publication June 1, 1977; accepted October 26, 1977.) 